\title{
Les néologismes par emprunt dans (le Monde et Al Ahram Hebdo) de 2011 à 2017
}

\section{Muhammad Ali Hefni Muhammad}

- Introduction:

L'emprunt est avant tout la qualité linguistique qui sert véritablement à exprimer une expérience culturelle, sociale, économique ou religieuse spécifique non seulement d'une culture donnée mais aussi de plusieurs cultures en même temps. Les locuteurs mondiaux utilisent des termes de leurs langues maternelles, tenant compte de toutes les caractéristiques de leurs régions dans le système linguistique de la langue parlée. Dans ce moment-là, ils ne cessent d'essayer de suivre les règles de la langue d'accueil. Avant de commencer cette recherche, nous devons proposer nombreuses définitions de l'emprunt :

Dans Le petit Robert, l'emprunt se définit comme :

« un acte par lequel une langue

accueille un élément d'une autre langue, élément "mot - tour" ainsi incorporé. Emprunt à l'anglais - anglicisme. Le fonds primitif et les emprunts- emprunt assimilé, francisé, traduit calque ».

Le dictionnaire de linguistique représente une définition stricte:

«Il y a emprunt linguistique quand un parler (A) utilise et finit par intégrer une unité ou un trait linguistique qui existait précédemment dans un parler (B)

${ }^{(*)}$ Cette recherche fait une partie d'une thèse doctorat, intitulée: " Les néologismes dans les deux journaux "Le Monde et Al - Ahram Hebdo " de 2011 à 2017 Etude linguistique", Sous la direction du. Dr. Hazem Ali Kamal El-Din - Faculté des Arts - Université Sohag \& Dr. Taha Rushdi Taha - Faculté d'Al-Alsun - Université Kafr El Sheikh.

$\left.{ }^{1}\right)$ Nouveau Petit Robert, Dictionnaire alphabétique et analogique de la langue française, version 2.1. Le CD-Rom, 2001 
(dit langue source) et que A ne possédait pas ; L'unité ou le trait emprunté sont eux - mêmes qualifiés d'emprunts. »

Pour résumer l'une des définitions les plus connues de l'emprunt, il se résume dans le processus dont le locateur d'une langue donnée emprunte une définition, un terme, une expression ou un trait linguistique (lexical, sémantique, phonologique, syntaxique) d'une autre langue (langue étrangère).

\section{- fatwa}

- Les emprunts dans notre corpus:

Seulement voilà : pour prétendre à l'investiture dans le Parti républicain d'aujourd'hui, M. Romney a dî se renier totalement. Il s'est conformé à ce que The Economist appelle une "liste de fatwas" composant le nouveau credo républicain.

Le Monde. Publié le 5 janvier 2012

Mohamad Saïd Raslane, directeur de l'institut Al-Forqane, a promulgué une fatwa selon laquelle "l'armée égyptienne a le droit et la responsabilité de combattre les takfiris parmi les Frères musulmans et les autres courants islamistes

\section{Al Ahram hebdo, le 17 juillet 2013}

Fatwa : c'est un nom arabe signifiant la décision du mufti sur un point de la loi des musulmans.

- chaâbi

Dès la fin des années 2000, le mouvement musical «mahraganat»ou électro-chaâbi s'est développé depuis les 
quartiers populaires, sans le contrôle des maisons de disques.

Le Monde. Publié le 25 novembre 2016

Chaâbi : c'est un nom utilisé dans le dialecte algérien et marocain pour désigner un genre musical qui s'est développé en opposition à la musique noble et savante.

\section{harraga}

C'est l'Espagne notamment, confrontée à une hausse générale des arrivées de migrants sur ses côtes cette année, qui a tiré la sonnette d'alarme. Les bateaux de harraga("ceux qui brûlent les frontières ») se sont entre autres multipliés dans la région de Murcie.

\section{Le Monde. Publié le 6 décembre 2017}

Harraga: c'est un mot d'origine arabe, c'est un nom donné aux immigrants illégaux du Maghreb, auxquels s'ajoutent de nombreux immigrants d'Asie. Le fait que les ces personnes migrent dans des bateaux simples est généralement dû à la difficulté à obtenir un travail décent, ce qui les a conduits à des solutions radicales. Les mauvaises conditions économiques et le chômage restent la principale raison pour laquelle les jeunes ont recours à cette action.

- Achoura

Les musulmans chiites célèbrent l'Achoura

PORTFOLIO Lundi 5 décembre, les chiites du monde entier célébraient le premier jour de la fête de l'Achoura qui culminera mardi, pour le 10 du mois de Moharram, avec des célébrations marquant la mort de l'imam Hussein.

\section{Le Monde. Publié le 5 décembre 2011}

Achoura: ce terme emprunté de l'arabe qui est " le $10^{\mathrm{e}}$ jour du mois de mouharram" le jour où Dieu a survécu à 
Moïse de Pharaon, c'est un évènement religieux des pays musulmans.

\section{- Al-Qaëda}

Abdallah Azzam, membre de

l'Organisation internationale des Frères

fondée vers la fin des années 1970, n'était-il

pas le parrain et le fondateur d'Al-Qaëda?

Al Ahram Hebdo. Publié le 4 Septembre 2013

Al_Qaèda: c'est mot arabe signifiant "la base" et désignant un mouvement djihadiste qui a été dirigé par Ayman al-zawshiri qui a suit Oussama Ben Laden après avoir été tué.

- waqfs

Le choix de ces 5 finalistes, parmi 17 candidats au départ, a été assuré par un comité électoral formé de représentants ecclésiastiques et lä̈ques du saint-synode, de l'organisme des waqfs (biens fonciers) coptes et du conseil communautaire copte.

Al Ahram Hebdo. Publié le 17 octobre 2012

Waqfs : Mot arabe désignant des biens inaliénables dont l'usufruit est consacré à une institution religieuse ou d'utilité publique.

\section{- Al Bedaya}

Dans un autre article de Hatem Hafez dans le tout nouveau journal en ligne AlBedaya, l'auteur réagit aux accusations contre les Frères musulmans d'avoir envoyé des milices sur la place Tahrir pour attaquer les manifestants.

Al Ahram Hebdo. Publié le 17 octobre 2012

Al Bedaya : Un nom arabe signifie le début et c'est un célèbre journal égyptien indépendant

- hashtag

Comme d'autres réseaux sociaux, Twitter a publié, dimanche 4 décembre, la 
liste des principaux mots clés - les hashtags

- et tendances des tweets de l'année 2011.

Publié le 07 décembre 2011

Hashtag : c'est un mot emprunté de l'anglais, le hashtag est un type de balise de métadonnées utilisé sur les réseaux sociaux tels que Tweeter et se fait en utilisant le \# tag qui permet aux gens de trouver facilement des messages concernant un sujet ou un contenu particulier.

- Shebab

«Le commandement militaire des moudjahidine shebab a ordonné un retrait tactique de Kismayo », a déclaré samedi le porte-parole des shebab, Ali Mohamoud Rage, à la suite du repli des shebab $d u$ Kismayo, le grand port du sud, qui représente leur dernier bastion important en Somalie.

Al Ahram Hebdo. Publié le 3 octobre 2012

Shebab: c'est un mot arabe qui veut dire "les jeunes" en français.

\section{- Djihadiste}

"Le mot Islam, il faut l'assumer, lui redonner sa grandeur, il faut le porter, il ne faut pas le laisser aux djihadistes », ajoutet-elle.

Al Ahram Hebdo. Publié le 26 septembre 2012

Djihadiste: mot arabe signifiant celui qui se rapporte du djihad. Djihad, C'est l'ensemble des devoirs religieux de musulmans qui ont pour but de s'améliorer à titre personnel et d'améliorer la société islamique.

\section{- Ennahda}

Les manifestants sont redescendus dans les rues pour réclamer du travail et ont 
appelé à la chute du gouvernement dirigé par les islamistes du parti Ennahda.

\section{Al Ahram Hebdo. Publié le 3 octobre 2012}

Ennahda : c'est un mot emprunté de l'arabe. C'est le mouvement historique représentant le mouvement islamiste en Tunisie, fondé en 1972 et officiellement proclamé le 6 juin 1981. Le mouvement n'a été reconnu comme parti politique en Tunisie que le 1er mars 2011 par le deuxième gouvernement de Mohamed Ghannouchi après le départ du président Zine El Abidine Ben Ali après la révolution tunisienne du 17 décembre 2010

- Khawaja

Cet extrait de son roman écrit en français et intitulé Khawaja révèle une sobriété et une retenue dans le style.

\section{Al Ahram Hebdo. Publié le 26 septembre 2012}

Khawaja : C'est un mot emprunté de l'arabe qui est un surnom donné à un homme occidental ou étranger.

- Hezbollah

Téhéran et Damas forment un axe régional destiné à faire face à l'hégémonie occidentale et basé sur le soutien au Hezbollah libanais et aux mouvements palestiniens radicaux comme le Hamas.

Al Ahram Hebdo. Publié le 3 octobre 2012

Le Hezbollah : c'est un terme arabe qui se compose de deux mots ( hezb + Allah) qui est un groupe chiite islamique armé et un parti politique basé au Liban.

- kholea

En 2000, le droit au kholea, qui permet à la femme de divorcer si elle abondonne ses droits pécuniaires à son mari, fut un progrès notable.

Al Ahram Hebdo, publiée le 17 octobre 2012. 
Kholea, C'est un mot arabe qui est une règle selon laquelle la femme peut divorcer de son mari sans avoir à donner d'explications en contrepartie d'une remise de sa dot.

- Nour

Chouq (envie), en attente du retour de son mari, s'adonne aux désirs de son corps et à la séduction des hommes. Nour (lumière),

Al Ahram Hebdo, publiée le 26 septembre 2012.

Nour, C'est aussi un terme arabe qui signifie la lumière par toutes ses sortes soit venant d'une source électrique soit de la nature.

\section{- feloul}

Les habitants saluent les manifestants et scandent avec eux les slogans. Difficile de préciser l'appartenance politique de cette masse d'Egyptiens. Des forces révolutionnaires? Des vrais ? De gauche? Des feloul? Peu importe.

\section{Al Ahram Hebdo. Publié le 3 juillet 2013}

feloul: C'est un terme politique arabe qui réfère aux partisans du président Mubarak.

- Tamarrod

Des activistes du mouvement Tamarrod (rébellion) profitent des rassemblements pour collecter des signatures sur leurs bulletins,

Al Ahram Hebdo. Publié le 26 juin 2013

Tamarrod: mot d'origine arabe, c'est un mouvement politique très célèbe dans la politique égyptien. Ce mouvement était à la tête des partis qui ont lutté la poltique de président Morsi. Le mot veut dire en français "rébellion".

- Al-Arraf 
Al-Arraf (le voyant), écrite par Youssef Maati et réalisée comme de coutume par son fils Rami Imam.

\section{Al Ahram Hebdo. Publié le 3 juillet 2013}

Al-Arraf : C'est un terme concernant la culture arabe. il donne le sens de (le voyant).

\section{- Daéya}

à travers le scénario de son nouveau feuilleton Al-Daéya (le prédicateur), réalisé par le jeune Mohamad Gamal AlAdl, et joué par Hani Salama et Basma.

\section{Al Ahram Hebdo. Publié le 3 juillet 2013}

Daéya : C'est un terme arabe qui signifie "un prédicateur". Il est celui qui parle publiquement des choses de Dieu aux non-croyants et enseigne les croyants (on dit prêcher). Le prédicateur est celui qui délivre un sermon dans un contexte religieux ou non.

\section{- Hazemoun}

Néanmoins, un autre nombre de formations islamistes, y compris les partis salafistes Al-Nour et Al-Watan, ainsi que le mouvement Hazemoun.....

\section{Al Ahram Hebdo. Publié le 3 juillet 2013}

Hazemoun : C'est un terme arabe politique et religieux en même temps. Il signifie (les partisans de l'ancien candidat salafiste à la présidence Hazem Salah Abou-Ismaïl).

\section{- Ikhwan online}

Le site de la confrérie des Frères musulmans Ikhwan online rapporte les propos de Assem Abdel-Magued, député au Sénat et membre de la Gamaa islamiya.

\section{Al Ahram Hebdo. Publié le 3 juillet 2013}

Ikhwan online : C'est un terme arabe qui donne le sens de "frères musulmans en ligne".

- baltaguis 
Selon certains avis, des parties inconnues auraient embauché des baltaguis pour créer le chaos à l'intérieur de la manifestation et attaquer l'ambassade et les forces de sécurité.

\section{Al Ahram Hebdo. Publié le 26 septembre 2012}

Baltaguis : mot arabe qui fait référence à celui qui pratique un type d'activité criminelle qui impose le contrôle, la terreur et l'intimidation des individus par la force en les agressant et parfois en les tuant dans le but de voler ou de supprimer l'opinion, de bloquer les routes et de brûler des bâtiments .

\section{- Chabihas}

Ceux-ci jouent aussi le rôle d'agents de sécurité, car ils auraient appris que des chabihas (hommes de main) seraient venus de Syrie pour semer le chaos.

\section{Al Ahram Hebdo. Publié le 26 septembre 2012}

Chabihas: C'est un terme utilisé en Syrie qui peut initialement faire référence aux gangs et les personnes qui ont utilisé la violence et les menaces d'armes pour servir une personne puissante afin de faire chanter et de terroriser les gens.

\section{- Tarawih}

Selon un décompte de l'AFP réalisé à partir de bilans de plusieurs organisations de défense des droits de l'homme, l'armée et les forces de sécurité ont tué 140 civils dimanche dans tout le pays, dont une centaine à Hama, et 24 autres lundi, dont dix ont été tués lundi à l'issue de la prière des Tarawih - prière de rupture du jê̂ne au premier jour du ramadan

Le Monde. Publié le 2 août 2011 
Tarawih : c'est un mot emprunté de l'arabe, qui signifie la prière musulmane que les musulmans font après la prière du soir (al-isha) dans le mois ramadan.

- Salafi

«Je suis salafi, mais je ne suis pas Daech, je suis contre Daech. J'ai même participé à une réunion avec Dounia Bouzar [médiatique chercheuse sur la radicalisation] », a argumenté la jeune fille vêtue d'un jilbeb prune et de gants noirs.

Le Monde. Publié le 4 octobre 2016

Salafi: c'est un mot arabe, celui qui suit le démarche du salafisme qui est le nom d'une approche qui appelle à comprendre le Coran et Sunna, suivant le démarche du prophète Mohamed et ses compagnons en tant que représentant de la religion de l'Islam.

- Etmarrad

Etmarrad (révolte-toi) est une chanson composée et chantée par Walid Saad.

Al Ahram Hebdo. Publié le 10 juillet 2013

Etmarrad: c'est un terme emprunté de l'arabe, C'est une chanson composée et chantée par Walid Saad. Il signifie " (révolte-toi). Tous ces termes sont nouveaux et nés lors de la révolution égyptienne de Janvier 2011.

\section{- Keswa}

Deux villes se juxtaposent, mais elles s'unissent au cours de plusieurs occasions de l'année. Celle du Mahmal, ce cortège religieux qui transfère la keswa.

\section{Al Ahram Hebdo. Publié le 3 juillet 2013}

Keswa : C'est un terme arabe qui signifie la couverture de la Kaaba en Arabie).

- Mouleds 
Les divertissements des habitants sont basés sur les célébrations sociales comme les noces de mariage, sans oublier les festivités religieuses comme les mouleds des saints musulmans ou chrétiens.

Al Ahram Hebdo. Publié le 3 juillet 2013

Mouleds: C'est un terme arabe pluriel qui signifie "les anniversaires" où les égyptiens font des fêtes pour célébrer les grands hommes de religion.

\section{1- Les différentes catégories de l'emprunt:}

Ce sont plusieurs critères qui sont pris en considération lors de la sous catégorisation de l'emprunt. Une véritable différence entre toutes les sortes et les catégories de ces critères qui montre la spécificité de chaque type selon des conditions particulières.

\subsection{Catégorisation selon la vivacité de l'emprunt}

L'emprunt est un grand et véritable phénomène linguistique et social qui suscite et provoque les notions de bilinguisme, d'inter-culturalité et d'interférence. L'emprunt- par ses deux catégories les plus célèbres, est à la tête des processus linguistique qui ont donné une certaine légitimité à la plupart des termes étrangers et bizarres.

Concernant l'emprunt en français ou le terme emprunté, il est utilisé principalement pour répondre à un besoin, c'est-àdire en vue de combler un vide dans la langue qui l'adopte car cette langue peut être incapable de formuler le sens correct bien voulu. D'ici vient la nomination un emprunt "nécessaire ou dénotatif". Cette catégorie de l'emprunt est souvent équivalente d'un néologisme ou d'une création de mot.

Quant à la deuxième catégorie la plus important aussi, c'est l'emprunt superflu, cette catégorie de l'emprunt se caractérise par l'existence d'un équivalent considérable dans la langue emprunteuse. C'est pour cette raison, un nombre des linguistes considèrent cette sorte comme un emprunt de "luxe". 
D'après le tableau, on constate que l'emprunt superflu est beaucoup d'emprunts. Cependant, l'emprunt superflu se manifeste plus au moins important, ce type a comme norme d'emprunter un nom nécessaire.

\begin{tabular}{|c|c|}
\hline Nécessaire & Superflu \\
\hline fatwa & bling-bling \\
\hline Achoura & Nesseitak \\
\hline Al-Qaëda & Khawaja \\
\hline feloul & Chouq \\
\hline Googliser & Surge \\
\hline Tarawih & Baladi \\
\hline waqfs & Al Bedaya \\
\hline hashtag & Nour \\
\hline
\end{tabular}

Nous avons pris un critère pour identifier l'emprunt nécessaire, ce critère consiste à rassembler tous les mots qui ont un enjeu technique ou scientifique, mais aussi, toute une nouvelle réalité ou une réalité existante dans une autre langue étrangère et que la langue emprunteuse veut l'adopté pour refléter une réalité étrangère et nouvelle qui elle-même empruntée ou adaptée.

\subsection{Catégorisation selon la formation de l'emprunt}

En ce qui concerne la catégorisation selon la formation de l'emprunt, Il y a trois catégories. Ce sont l'emprunt lexical, l'emprunt syntaxique et l'emprunt phonétique.

1.2.1. L'emprunt lexical : Il y a l'emprunt lexical intégral qui met en considération la forme et le sens selon un traitement spécifique. Il y a aussi un emprunt partiel qui ne peut traiter qu'une seule chose soit le sens soit la forme. L'emprunt lexical porte sur le mot.

Selon Foudil Chériguen, l'emprunt lexical peut être défini comme : 
"Une unité de fonctionnement comportant une ou plusieurs parties, toutes susceptibles d'usage syntaxique autonome. Il est mis en morphologie (...) parce que passé dans la langue cible. La base lexicale relève de la langue source. Il y a donc un rapport avec celle-ci (rapport étymologique)... ${ }^{1}$

On distingue quatre types d'emprunts lexicaux.

a- L'emprunt intégral, dans cette sorte d'emprunt lexical qui traite comme nous l'avons déjà dit la forme e le sens, l'on ne fait jamais aucun changement ni graphique ni phonétique lors de la mise en considération de la forme et du sens. $\boldsymbol{E X}$ : week-end, staff, hold up, shopping.

b- L'emprunt hybride, c'est la catégorie de l'emprunt qui traite le sens à condition que sa forme ne soit pas intégrale. $\boldsymbol{E X}$ : Coach de vie.

c- Le Faux emprunt, c'est la forme intégral de l'emprunt qui se compose d'éléments formels empruntés surtout d'anglais, mais sous une forme française. Cette sorte représente un résultat d'emprunt limité dans sa forme. Jacquet-Pfau et Sablayrolles, mettent une définition de faux emprunts présentés dans le cas du français; ils sont des " créations françaises mettant en ouvre des formants d'origine étrangère et se conformant le plus souvent aux principes de la langue étrangère d'où sont issus ces formants $»^{2}$. EX : slip - tennisman - camping-car.

$\left.{ }^{1}\right)$ Foudil Cheriguen, Typologie des procédés de formation du lexique. Cahiers de lexicologie, numéro 55, 1989, pp.53-59.

${ }^{4}$ )- Jean-François Sablayrolles et Jacquet-Pfau Christine, « Les emprunts : du repérage aux analyses. Diversité des objectifs et des traitements », In Neologica $\mathrm{n}^{\circ} 2,2008$, pp. 19- 38. 
d- Le calque, cette sorte d'emprunt lexical est composée de trois catégories différentes

- Le calque morphologique.

- Le calque sémantique.

- Le calque phraséologique.

1.2.2. L'emprunt syntaxique : En définissant l'emprunt syntaxique, il est un emprunt qui a une structure syntaxique tout à fait étrangère. Cette sorte de l'emprunt qui s'agit de la structure de la phrase.

Il est facile de remarquer l'influence considérable de l'anglais qui se manifeste dans le choix de la préposition « sur» ou de la conjonction. EX : être sur l'avion insister que.

Cette sorte de l'emprunt est très claire dans les situations bilinguisme social ${ }^{\mathbf{1}}$

1.2.3. L'emprunt phonétique : Cette catégorie d'emprunt traite et concerne particulièrement la prononciation étrangère. $\boldsymbol{E} \boldsymbol{X}$ : le mot «gym » en anglais se prononce [djim ] mais en français [ Zim ].

\subsection{Le classement des emprunts selon les différentes}

\section{langues :}

Les sources des lexies sont la base essentielle qui définissent et précisent le classement des emprunts Mais "Il ne faut cependant pas confondre langue d'origine de la lexie et langue à laquelle la langue emprunteuse l'emprunte : certaines lexies passent par plusieurs langues. Jungle est un emprunt par le français à l'anglais d'un mot indien jongola. Thé est un mot chinois malais passé par l'intermédiaire du néerlandais (la forme thé vient du latin moderne 1657)... $»^{2}$.

\footnotetext{
$\left.{ }^{1}\right)$ Le bilinguisme social met l'accent sur les forces linguistiques qui existent dans une communauté ou dans un groupe ethnique. Le chercheur est amené à s'interroger sur le degré de relation entre les forces politiques, sociales, éducatives, culturelles et les forces de la langue.

$\left.{ }^{2}\right)$ A Queffelèc et al, (2002), Le français en Algérie : Lexique et dynamique, Louvain-LaNeuve, De Boeck-Duculot-AUF, p.126
} 
C'est pour cette raison et en ce qui concerne notre corpus, les sources linguistiques auxquelles les lexies sont empruntées se répartissent comme suit :

\section{3.a. L'emprunt à l'arabe :}

Comme nous le constatons toujours, l'emprunt dans le français occupe une place très importante et très efficace non dans l'histoire de la langue. De l'autre côté, les lexies d'origine arabe "soit dialectal soit standard", anglais et allemand sont aussi d'une importance et d'une efficacité inévitable. L'emprunt à l'arabe dialectal est en tête et sur toutes les autres considérations. Ces emprunts enregistrent et dénotent une certaine « réalité » notamment spécifique à la société égyptienne, c'est-à-dire ignorés des locuteurs natifs de français ou même parfois ignorés par d'autres locuteurs. L'emprunt à l'arabe se représente comme un code spécifique que seul un lecteur égyptien (ou arabe) peut déchiffrer, et qui échappe aux autres lecteurs francophones.

En consultant Al-Ahram-Hébdo, nous pouvons déduire que les emprunts à l'arabe "littéraire /dialectal" sont en première position. La forte représentation de cet ensemble est liée à l'identité des créateurs et des lecteurs qui sont tous des locuteurs égyptiens ayant pour langue maternelle la langue arabe. Il est bien remarquable de noter l'importance et la croissance des mots empruntés à l'arabe. Ces innovations lexicales à travers l'emprunt semblent répondre à des besoins de communication et ils évoquent des phénomènes sociaux et culturels. Ils servent donc nécessairement à exprimer la réalité socioculturelle égyptienne. Cette importance n'est peut être négligée puisque s'il n'y avait pas cette nécessité d'emploi, l'emprunt n'existerait pas. D'autre part, nous trouvons que certains emprunts n'ont pas et ne peuvent pas avoir un équivalent en langue française à cause de la différence culturelle qui se montre parfois dans quelques cotés. C'est le cas des termes empruntés surtout au domaine religieux. Nous avons recensé l'emprunt Waqfs dans : l'organisme des waqfs. Salafiste, tarawih. 
Alors, certains emprunts peuvent avoir des synonymes en langue française mais selon $Z$. Lafage cet équivalent peut être « un mot $[. .$.$] peu précis,...peu satisfaisant car il est souvent$ ambigu ${ }^{l}$ et ne reflète que la réalité désignée de façon imparfaite. En prenant comme exemple, nous relevons l'exemple de l'emprunt "harraga" qui est un mot populaire introduit pendant ces dernières années. On doit ajouter qu'il s'agit aussi d'un néologisme de l'arabe dialectal, crée du verbe « brûler ». Ce néologisme est bien utilisé pour decrire particulièrement les bateaux maghrébins qui envahissent clandestinement les frontières pour atteindre les côtes européennes. Mais, on remarque que l'équivalent en français n'exprime pas exacte au point qu'il révèle la lexie en arabe. De plus, le terme "harraga" est considérablement connoté, il est employé pour livrer une dimension sociopolitique et socioculturelle aux situations complexes auxquelles sont confrontés les jeunes maghrébins.

Les exemples sont véritablement nombreux que le chroniqueur recourt. En commençant par le terme spécifique "moudjahidine "ou encore l'emprunt "chouhada" Ces deux emprunts servent en effet à relever moins d'un besoin de communication, puisque les termes équivalents "combattant" pour le premier et "martyr" pour le second existent en langue française, mais en optant pour moudjahidine ou chouhada, le chroniqueur surcharge sémantiquement son texte dans la mesure où ses emprunts impliquent le sacrifice de soi au nom de Dieu, et ce sont des notions fortes et culturellement riches en signification et que, ce faisant, le rédacteur de la chronique ramène en fait à la mémoire de son lecteur, tout un système de valeurs culturelles et religieuses qui, en quelque sorte, assignent aux termes empruntés une charge sémantique dépassant celle que leur attribue le contexte dans lequel ils se réalisent.

$\left.{ }^{1}\right)$ LAFAGE, Suzanne, Français écrit et parlé en pays éwé (Sud-Togo), Paris, CNRS -SELAF. 1985, p.487 
Beaucoup d'innovations lexicales représentent le phénomène d'emprunt à l'arabe standard comme le terme "achoura", etc. Spécifiques à l'univers référentiel de la religion et de la civilisation arabe et sont communs à la communauté islamique.

D'après ces données, nous pouvons affirmer que le taux de recours aux emprunts à l'arabe montre que le conflit linguistique français/arabe est bien réel. Mais ce conflit ne se situe pas essentiellement entre les deux langues académiques (arabe littéral / français) comme le déclarent A Queffélec et al, qui pour eux, chacune de ces deux langues essaye de monopoliser les atmosphères de contact et d'accaparer le plus de domaines d'emploi. Dans les journaux, ce conflit se situe entre le français et $l^{\prime}$ arabe dialectal ${ }^{1}$.

Nous constatons que le recours à l'emprunt montre que les créations vernaculaires restent amplement favorisées d'une manière qui montre une véritable valeur. Cela montre également que cette langue vernaculaire intervient considérablement et reste largement privilégiée même s'il s'agit d'un journal francophone où la situation de communication est formelle. Or, nous pensons que ces emprunts ne représentent aucune menace sur la structure de la langue emprunteuse ou d'accueil, c'est-à-dire, la langue française.

1.3.a.1. L'intégration des emprunts du français à l'arabe:

Nous avons affirmé que l'emprunt linguistique qui se considère comme un procédé d'une nature néologique occupe une place si efficace dans le discours journalistique et il apparaît également dans tous les niveaux de langue; "langue soutenue, langue courante, dialecte, etc". D'autre part, il est nécessaire pour nous de voir et d'essayer de montrer comment ces mots étrangers s'intègrent linguistiquement dans un autre

$\left.{ }^{1}\right)$ Queffélec, A., Y. Cherrad-Benchefra, Y. Derradji., V. Debov et D. Smaali-Dekdouk., 2002. Le français en Algérie lexique et dynamique des langues, Louvain-La-Neuve, De Boeck-Duculot-AUF. P. 140. 
système puisque. Ainsi, l'acte ou le processus d'emprunter un terme est dans la plus part de temps, suivi d'adaptation dans son nouvel paysage linguistique. Dans cette partie, nous tentons de mettre en relief la réponse de la question suivante: Quels sont les critères et les degrés d'intégration de l'emprunt dans la langue d'accueil?

Selon L. Guilbert (1975), Ce sont trois critères auxquels dépend l'installation d'un terme étranger dans le système linguistique d'une langue d'accueil: paramètres phonologique (et graphique), morphosyntaxique et sémantique. En ce qui concerne la langue prêteuse, c'est-à-dire l'arabe, il présente plusieurs différences par rapport au français. Ce qui oblige les chercheurs et linguistes, dans beaucoup de cas, à adapter les termes locaux aux exigences de prononciation et de fonctionnement des mots français généralement.

Donc, ces trois critères montrent un rôle sans précédent dans l'adaptation juste des emprunts dans leur nouvel environnement et atmosphère linguistique car le mot étranger ne sera admis sauf s'il est adapté dans l'usage de la langue d'accueil et qui va se varier selon différents critères d'intégration qui dominent l'usage de la langue d'accueil.

a- Intégration phonologique et phonétique:

Dans le cas de l'emprunt, ce critère semble très pertinent et très particulier puisque ce dernier est toujours transféré d'un système phonologique à un autre système tout à fait différent. Il faut insister que l'adaptation phonique est inévitable quand il s'agit de l'emprunt du mot étranger à cause des différences entre les systèmes phonologiques de deux langues concernées arabe et français

Cependant, l'emprunt lexical est caractérisé toujours par sa puissance de conserver les traits phonologiques de la langue d'origine. C'est pourquoi, les trois hypothèses ont été établies concernant l'intégration phonique des lexies empruntées.

Pour A. Queffelèc, la première hypothèse précisent l'intégration totale des lexies empruntées dans le système 
phonologique de la langue cible. D'ailleurs, dans la lexicologie traditionnelle, ce type d'intégration représente "l'un des critères les plus pertinents pour différencier mots naturalisés (dont la forme sonore respecterait le système de la langue emprunteuse) et simples citations (conservant phonétiquement les traits de la langue d'origine).» ${ }^{l}$ Dans ce cas, nous recourons à une catégorie d'emprunts où le critère de degrés d'intégration au système phonologique est mis en considération.

Deroy a montré que "la première adaptation que subit un mot emprunté est phonétique et, dans une certaine mesure, phonologique.» Mais dans la plupart de cas, nous trouvons que l'intégration phonologique est accompagnée par l'intégration graphique.

Mais, nous voyons, comme tant d'autres chercheurs qui ont mené de vastes études dans ce domaine de l'emprunt, que cette sorte d'intégration phonique des mots étrangers, d'une manière générale estime et traite le système phonique de la langue d'origine et qu'il est conforme sans cesse au système phonique de la langue d'accueil. Ainsi, les lexies empruntées recensées dans notre corpus ne posent aucun problème car ces dernières, dans l'ensemble, ne présentent pas de particularité phonétique par rapport à la langue française et ils semblent poser moins de problème d'intégration au système de la langue.

Quant à la deuxième hypothèse, ce dernier axe, selon le même auteur, l'indépendance totale de la forme sonore " fait des sonorités" des lexies empruntées vis-à-vis du système phonologique de la langue d'arrivée. D'où la conservation des sons propres des phonèmes ainsi que leur représentation graphique qui reste conforme à la langue d'origine.

Ainsi, pour la lexie niqab, qui est un mot tout à fait religieux et qui signifie "long voile islamique couvrant le visage d'une femme, à l'exception des yeux", le chroniqueur a conservé

$\left.{ }^{1}\right)$ QUEFFÉLEC A. (1997). Le français en Centrafrique. Lexique et société, coll. "Universités francophones", Vanves : EDICEF, p.1- 9.

$\left.{ }^{2}\right)$ Louis Deroy, l'emprunt linguistique, Collections - Séries -Revues, 1956, p. 235 
la présentation graphique du phonème [q]. Par contre le phonème $[\mathrm{k}] \mathrm{n}$ 'a pas été utilisé pour substituer le son [q] et ainsi de suite.

En ce qui concerne la troisième et la dernière hypothèse, il existe véritablement un chevauchement complétif entre le système phonologique de la langue d'origine et celui de la langue d'accueil. Concernant notre corpus, ce chevauchement a été bien défini lors de la collection des lexies empruntées. En effet, il arrive très souvent qu'une double prononciation s'installe, l'une conforme à la langue française et l'autre correspond au système phonologique de la langue d'origine. Dans ce cas, il s'agit du son $[\varepsilon]$ prononcé et orthographié différemment parfois « a » ou « à » dans les lexies empruntées recensées, aïd, chaâbi, etc.

En ce qui concerne des emprunts français à l'arabe, l'épreuve de notre corpus révèle également qu'un véritable nombre d'emprunts conservent dans la langue d'accueil la phonétique originelle de la langue d'origine.

\section{b- Intégration graphique:}

L'emprunt impose un autre critère tout à fait considérable, celui de la transcription phonétique des phonèmes linguistiques. Ce critère graphique accompagne souvent le premier, ils sont fortement liés l'un à l'autre car le critère phonologique mène momentanément à la standardisation orthographique de l'emprunt intégré.

L'intégration graphique, de sa part, peut devenir un bon indicateur ou un bon axe d'adaptation aux normes graphiques de la langue d'accueil. La plupart des emprunts célèbres sont d'une graphie tout à fait unifiée.

Alors, l'emprunt peut montrer de nouvelles graphies plus ou moins conformes et justes à celles de la langue source. Ce cas peut se produire lorsque la langue d'accueil fait recours à un code complètement ou partiellement "commun" que celle d'origine. C'est l'exemple des langues européennes qui s'entrent dans des relations entre elles ou avec les autres 
langues anglo-saxonnes qui ne demandent généralement aucune modification de la forme graphique du mot emprunté. Or, d'un point de vue graphique, l'on peut déduire que "l'emprunt à l'arabe est généralement peu intégré". Ceci dit qu'une hésitation au niveau de la graphie du mot étranger peut se produire. A ce propos, Y Derradji précise que "l'écart qui sépare la phonie arabe de la phonie française pour certains sons est tel que l'adoption est rendue difficile par une prononciation et une graphie très souvent fautives. " ${ }^{l}$ Généralement, pour la francisation "l'acte d'entrer un terme en français" de ces phonèmes, la tendance dominante est de les remplacer par des sons proches qui existent déjà en français.

Or, nous insistons sur le plan graphique qu'un nombre important de lexies empruntées ont une transcription qui n'est jamais stable. Ceci dit que l'intégration est encore en cours. Ainsi, cette adoption de multiples formes graphiques montre véritablement l'instabilité de la forme graphique faite par une immense frontière entre les systèmes graphiques du français et de l'arabe. Pour d'autres lexies, la graphie ainsi que la phonie sont établies d'une manière qui explique que leur processus d'intégration dans la langue d'accueil est bien complet.

Pour notre étude, l'acte d'intégration est, à notre sens, très essentiel car nous nous attachons à la nature graphique de notre corpus et la manière dans laquelle les phonèmes des lexies empruntées sont transcrits. Ainsi, pouvons -nous remarquer conformément au propos de D Morsly, que le chroniqueur EL Guellil a adopté divers procédés graphiques pour transcrire ces graphèmes arabes en faisant appel à son « imaginaire graphique ». Ceci montre donc l'instabilité de la forme graphique et

$\left.{ }^{1}\right)$ Yacine Derradji, LE FRANÇAIS EN ALGÉRIE : LANGUE EMPRUNTEUSE ET EMPRUNTÉE, Université de Constantine, 1999, http://www.unice.fr/bcl/ofcaf/13/derradji.html. Consulté le 12 novembre 2018. 
représente aussi de grandes différences qui existent entre le système graphique français et le système graphique arabe ${ }^{1}$.

Concernant le phonème [q], la transcription de ce dernier est instable. Nous constatons qu'il existe deux orthographes différentes. Le « $\mathrm{k} »$ pour la lexie empruntée «takfiris» et le « q » pour le mot «Al-Qadr» ou «Chouq». Nous avons pu montrer un autre phonème, c'est le [w] dont la graphie est « w » nous avons constaté, par exemple qu'il est transcrit en « ou »dans mouleds. Tandis que pour l'emprunt tarawih, le phonème [w] est orthographié par « w ».

\section{c- Intégration morphosyntaxique :}

Pour l'intégration des emprunts, il y a un autre critère que l'on doit mettre en considération. C'est un très pertinent critère qui s'impose sans cesse dans les études linguistiques. Cette intégration est bien remarquée lors du processus dérivationnel morphologique qui affecte massivement les emprunts.

- Les intégrations dérivationnelles:

Selon L. Guilbert, cette sorte de l'intégration dérivationnelle est concrétisée quand «un mot étranger, dès le moment où il sert de base à une dérivation selon le système morphosyntaxique de la langue française est véritablement intégré à notre langue $\|^{2}$. Les exemples déjà cités montrent l'importance de la formation de nouvelles lexies telles que djihad qui a donné djihadistes, salafi a donné salafistes et le verbe Etmarrad est le dérivé du substantif Tamarrod.

En analysant tout ce qui a précédé, nous déduisons que les chroniques de la presse jouent, du point de vue linguistique, un véritable rôle puis qu'elles assurent encore l'intégration des termes arabes au système de dérivation du français, et cela en mêlant le radical de l'emprunt et l'affixation du système français.

$\left.{ }^{1}\right)$ D. Morsly, "Génération M6, le français dans le parler des jeunes algérois", Plurilinguismes, 12, 1996, pp. 111-122

$\left.{ }^{2}\right)$ Louis Guilbert, La creativité lexicale. Paris: Librairie Larousse, 1975. P. 97. 
- Les intégrations compositionnelles :

Pour cette catégorie de l'intégration, l'intégration compositionnelle peut montrer à son tour deux cas de formation : la première est la composition nominale. Celle-ci est construite à partir d'un syntagme du type nom arabe plus caractérisant la langue française comme dans les exemples suivants:

Hezbollah libanais, Ikhwan online, à travers ces deux exemples bien illustrés, l'on peut avoir une composition à partir d'un syntagme de la langue d'accueil plus une lexie de la langue d'origine (français+arabe). Selon ce type, nous avons recensé les exemples suivants : parti Al-Moatamar, néo-Wafd, grand moudjahid, mouvement Hazemoun, feuilleton Al-Daéya, etc.

- L'adjonction du genre :

Théoriquement, la lexie empruntée conserve son genre dans la langue d'origine. D'autre part, les marques et les caractéristiques du genre dans l'emprunt à l'arabe sont conformes au système orthographique de la langue française. GAADI insiste sur la même conformité de l'emprunt et de son genre emprunté lui-même de la langue source ${ }^{1}$. De plus, la préservation du genre de la langue d'origine est consolidée par la détermination. D Smaali affirme que «le déterminant varie en fonction de la classe masculin/féminin à laquelle appartient le lexème en arabe» ${ }^{2}$.

Il est à noter que le genre de l'adjectif est le plus conforme et le plus apte aux règles d'accord de la langue d'accueil. Des lexies comme La Jamaa islamiya, (Le groupe islamique), Jabal Al-Halal (La Mont Al-Halal), Nass al-nahr (les gens de la

$\left.{ }^{1}\right)$ GAADI D. (1995). "Le français au Maroc. L'emprunt à l'arabe et les processus d'intégration", dans A. Queffélec, F. Benzakour et Y. Cherrad-Benchefra (éds), Le français au Maghreb, Aix-en-Provence : Pub. Université de Provence, pp. 131-151.

$\left.{ }^{2}\right)$ SMAALI D. , Les particularités lexicales du français dans la presse algérienne actuelle, Mémoire de maîtrise, Université de Provence,1994,p. 203. 
rivière), la dawla islamiya (état, nation islamique), etc. sont de expressifs exemples.

\section{- L'adjonction du nombre :}

De sa part, le nombre représente un autre facteur dans le contexte des emprunts. On peut dire que la diversité des marques du nombre des emprunts soutient que l'on distingue trois types dans le marquage du nombre de certains emprunts :

La première catégorie des lexies empruntées est conforme au système de la langue source, avec adoption de la forme du pluriel de la langue d'accueil. En d'autres termes, pour former le pluriel des mots empruntés, le chroniqueur préfère tout simplement respecter la forme morphologique de la langue française en ajoutant le «s », la marque du pluriel. Des lexies empruntées telles que les fatwas, les moudjahidines, des baltaguis, des chabihas, ont été recensées.

La deuxième catégorie unit les emprunts pour lesquels le chroniqueur fait apparaître la marque du pluriel de la langue arabe. Mais, en règle générale, ces emprunts sont adaptés par le système de déterminants de la langue d'adoption, cependant la détermination peut être réalisée à travers les marqueurs de la langue arabe: selon le genre de la lexie empruntée, on rencontre les déterminants masculin/féminin spécifique à la langue d'adoption : les moudjahidine, des shebab, des soukouk, Des feloul, des Ultras ahlawi ...etc sont des mots empruntés relevés lors de la collections des lexies.

Pour ces lexies, nous avons découvert plusieurs types de déterminations : Déterminant défini « la » dans la lexie empruntée la morabaha, la keswa ; déterminant défini « le » dans la lexie empruntée le Hamas; déterminant indéfini « un » dans un hashtag; l'emploi du déterminant défini arabe « al » dans : al-charia,, Al-Qaëda, Al-Wachm, Al-Arraf, etc.

Il faut montrer l'emploi d'autres morphèmes qui s'accordent fortement aux lexies empruntées mais qui sont véritablement rares. Les pronoms possessifs « son » et « sa » 
sont utilisés avec les lexies empruntées, comme niqab, pour former les syntagmes son niqab.

La dernière catégorie unit les emprunts qui sont caractérisées par les marques de deux systèmes linguistiques. Y Derradji précise que «l'emprunt reçoit les marques de pluriel de l'arabe avec "facultativement» la marque s du pluriel français : ainsi l'emprunt à la langue arabe est susceptible de recevoir le pluriel du système arabe et simultanément le pluriel du système français» ${ }^{l}$. Ainsi pour marquer le pluriel des emprunts tels que : fatwa, takfiri, bahaïe, le chroniqueur a respecté le système linguistique de la langue française en ajoutant tout simplement « $\mathrm{s}$ » pour avoir les lexies les fatwas, les takfiris les bahaï. Or, le pluriel de ces mots est bien disponible dans le système arabe : fatawat /fatwat, bahaïen. takfirien

Dans le cas d'autres emprunts, le rédacteur des chroniques journalistiques s'est servi du pluriel des deux systèmes arabe et français comme dans la lexie empruntée : le moudjahid / les moudjahidines. Donc le pluriel des lexies empruntées peuvent adopter une forme hybride.

\section{d- Intégration sémantique:}

Il faut montrer avant tout que les lexies empruntées conservent généralement leur sémantisme dans leur langue d'origine. Mais, il arrive parfois que "l'emprunt d'un mot entraine aussi parfois des modifications sémantiques $»^{2}$. Ces modifications ou cette néologie sémantique, comme le postule D Morsly, est « déterminée soit par le contexte social, soit par le contexte syntaxique $»^{3}$. Cette règle est effectué lorsqu'un emprunt est intégré dans une langue d'accueil peut générer

$\left.{ }^{1}\right)$ Yacine Derradji, LE FRANÇAIS EN ALGÉRIE : LANGUE EMPRUNTEUSE ET EMPRUNTÉE, Université de Constantine, 1999, http://www.unice.fr/bcl/ofcaf/13/derradji.html. Consulté le 25 decembre 2018

$\left.{ }^{2}\right)$ DEROY L. (1980). "Vingt ans après l'Emprunt linguistique: critique et réflexions", Cahiers de l'Institut de Linguistique de Louvain, 6, 1-2, p 261.

$\left.{ }^{3}\right)$ Morsly, D. (1988). Le français dans la réalité algérienne. Thèse de doctorat d'État, Université de Paris V. p.6. 
d'autres signifiés. Donc, un autre sens peut lui être attribué soit en modifiant son champ d'application initiale soit tout au moins élargi.

De tout ce qui a précédé, nous pouvons affirmer que ces emprunts gardent plus au moins une partie de leur caractéristique dans la langue d'origine. Ainsi, nous pouvons ajouter que ce recours et ce transfert massif des emprunts des langues locales vers le français en Algérie, n'est pas un phénomène nouveau.

En effet, le chroniqueur s'en sert pour des besoins de communication et partant d'accrocher l'attention de ses lecteurs.

" En effet, lorsqu'on transporte une langue en dehors de son aire d'origine, dans un milieu naturel et culturel différents, il faut s'attendre à ce que soit introduit dans son lexique un nombre important d'emprunts (et de calques) nécessaires pour satisfaire des besoins nouveaux d'expression et de communication. La proportion d'emprunts dans la presse locale semble être très importante, en raison, certainement, des différences très profondes entre le milieu emprunteur et le milieu d'origine.»" ${ }^{\text {. }}$

\section{3.b. L'emprunt aux autres langues :}

Dans le même contexte d'étude, l'importance des mots empruntés aux autres langues étrangères dans les chroniques journalistiques. Ce tas des mots construisent une matière fertile de l'emprunt. Ces lexies semblent répondre à des besoins de communication et ils évoquent des phénomènes étrangers.

$\left.{ }^{1}\right)$ Redouane S-E., « Les emprunts dans la presse marocaine d'expression française : problem d'intégration.» Disponible: http://www.docstoc.com/docs/81190373/doc_3_. Site consulté le 20/1/2019. 
En effet, un grand taux des néologismes empruntés au système linguistique anglais, comme fitst, come-back, ou encore le slow food, occupant ainsi la troisième position. D'un point de vue linguistique, l'emprunt à l'anglais s'explique par le fait que cette langue est très fertile surtout dans les éléments indiquant les qualités des choses et des gens. Ainsi, le chroniqueur emprunte à l'anglais confirmant le prestige dont bénéficie la langue anglaise.

En choisissant quelques autres exemples, nous prenons le perse "chicha", turque "döner kebabs", l'italien "stampa" ou encore la lexie russe nomenklatura. Il y a aussi des lexies empruntées à espagnol fiesta, des lexies d'emprunts en langue italienne comme "la camorra".

D'une part, l'emprunt pourrait s'interpréter comme un rejet de tout modèle social générateur. C'est en effet dans sa conscience de la dimension symbolique du langage que le chroniqueur se refuse, dans son opposition à un quelconque modèle, à recourir à la langue qui le véhicule. De ce fait, il y a une relation tout à fait intime entre la langue et " la chose ", comme l'écrit B. Malmberg en affirmant l'importance de cette question pour l'homme de culture moderne : "la relation qui existe entre la langue et la "chose " était dite SYMBOLIQUE (...) Et nous avons vu que c'est cette fonction qui, dans la communication de l'homme de culture moderne, occupe la première place ou qui, en tout cas, est généralement acceptée comme dans les sciences du langage. " 1.

Selon C. Fitouri dans son ouvrage consacré au biculturalisme " [...] Bien que, la nouvelle culture arabomusulmane jeune et vigoureuse sera dans sa phase ascendante une culture ouverte à toutes les influences. C'est ainsi qu'elle assimilera les cultures grecques, persane et hindoue, qu'elle s'ouvrira aux influences judaïques et chrétiennes et qu'elle

1) Malmberg B., (1979), Le langage signe de I'humain, Picard, collection Empreinte, Paris, pp.192-193. 
prendra des colorations aussi bien orientales qu'occidentales sans jamais se dissoudre dans ces divers éléments et sans bien sûr donner l'impression de l'amalgame ou de la mosaïque. N'est-ce pas là un bel exemple de synthèse des cultures, exemple qui n'a jamais cessé tantôt d'étonner, tantôt d'inquiéter. "1

\subsection{Classement des emprunts par domaine :}

Généralement, classer les emprunts d'une manière définitive dans un domaine ou dans un autre n'est pas facile. Comme le montre Ch. Marcellesi, Cet affaire d'appréciation personnelle ainsi que pour M.F Mortureux, devrait s'appuyer d'abord sur « l'intuition $»^{2}$. En outre, l'examen de l'emprunt dans le français en contexte égyptien présente un réseau de champs sémantiques qui se superposent et s'interpénètrent.

D'autre part, les néologismes par emprunts dans les domaines différents sont considérables. L'on ne peut jamais ignorer les spécificités -morphologiques et sémantiques qui permettent de les distinguer des mots plus usuels. De plus, il reste une remarque très efficace, c'est classer les néologismes par domaine. Cette faculté ou compétence implique qu'on ne peut se limiter au terme dans son isolement mais qu'on l'examine d'abord dans son contexte. Il faut montrer qu'un grand nombre des néologismes demeurent difficilement classables en termes de domaines. Il s'agit de délimiter les domaines dans lesquels apparaissent les emprunts que d'affirmer une quelconque exclusivité des néologismes par emprunts puisque ceux-ci peuvent en effet couvrir plusieurs domaines. Le classement par domaine doit être pris en considération comme suit:

$\left.{ }^{1}\right)$ Fitouri C., (1983), Biculturalisme, bilinguisme et éducation, Ed. Delachaux et Niestlé, p.22.

$\left.{ }^{2}\right)$ Mortureux M-F, (2011), " La Néologie Lexicale : De L'impasse à L'ouverture », In Langages $n^{\circ} 183$, p.14 
a- Emprunts attachés au domaine social :

Par la comparaison entre les domaines, la prédominance est véritablement attribuée aux néologismes par emprunts attachés au domaine social. Nous prenons comme exemple les emprunts suivants : Al-Masréyine Al-Ahrar , Fassad bel bélad, kholea, " madrasset al-mohandes khana ", Ennahda, Gheriyani,...etc. Cela justifie la diffusion des néologismes dans le corps social ${ }^{1}$. Vient ensuite le domaine de la culture qui peut unifier le sport, la musique, l'art culinaire, etc.

b- Emprunts attachés au domaine culturel:

S-J Bernard enregistre que "la langue n'existe pas séparément de la culture. En effet, la langue n'est qu'un aspect d'une culture ». La relation semble complétive entre les lexies et l'identité culturelle et sociale bien particulière.Dans le même contexte, nous ne pouvons ignorer les emprunts religieux surtout à révéler la civilisation arabo-musulmane, par exemple: salafistes, Al-Karama, tarawih, wahhabistes, Ansar Dine, charia, niqab, Jamaa ...etc. sont de bons exemples.

c- Emprunts attachés aux autres domaines:

Les emprunts sont mélangés fortement au domaine politique, technologique, scientifique, et économique. Ils restent relativement importants représentant ainsi des proportions faibles qui varient respectivement. Parmi les emprunts repérés, nous citons hashtag, hezb, i-phone, tweet, Baas ...

$\left.{ }^{1}\right)$ Grunig B-N, (2003), " Dynamique et lexique ", In L'innovation lexicale, Honoré Champion Éditeur, Paris, p.14 


\section{- Conclusion:}

A travers cette recherche, nous avons découvert que l'emprunt représente un facteur efficace dans l'étude des néologismes, que les genres de l'emprunt peuvent construire une véritable fortune linguistique, que la plupart des emprunts appartient à la langue arabe notamment dans le domaine politique grâce aux révolutions et mouvements politiques qui ont augmenté pendant les 10 ans derniers. En ce qui concerne les emprunts économiques, scientifiques et techniques, appartiennent à la langue et à la culture anglaise, puisque la langue anglaise représente la langue officielle de tout développement partout.

Finalement, nous avons trouvé que l'emprunt peut être existé relativement dans tous les domaines de la vie quotidienne. Les côtés culturels n'échappent pas d'être influencés par la compétence de l'emprunt. 


\section{Références:}

- Foudil Cheriguen, Typologie des procédés de formation du lexique. Cahiers de lexicologie, numéro 55, 1989, pp.53-59.

- Jean-François Sablayrolles et Jacquet-Pfau Christine, "Les emprunts : du repérage aux analyses. Diversité des objectifs et des traitements », In Neologica $\mathrm{n}^{\circ} 2,2008$, pp. 19- 38.

- A Queffelèc et al, Le français en Algérie : Lexique et dynamique, Louvain-La-Neuve, De Boeck-Duculot-AUF, 2002.

- LAFAGE, Suzanne, Français écrit et parlé en pays éwé (Sud-Togo), Paris, CNRS -SELAF. 1985.

- A Queffelèc. Le français en Centrafrique. Lexique et société, coll. "Universités francophones", Vanves : EDICEF,1997.

- Louis Deroy, l'emprunt linguistique, Collections - Séries Revues, 1956.

- Yacine Derradji, LE FRANÇAIS EN ALGÉRIE : LANGUE EMPRUNTEUSE ET EMPRUNTÉE, Université de Constantine, 1999 , http://www.unice.fr/bcl/ofcaf/13/derradji.html. Consulté le 12 novembre 2018.

- D. Morsly, "Génération M6, le français dans le parler des jeunes algérois", Plurilinguismes, 12, 1996, pp. 111-122

- GAADI D. "Le français au Maroc. L'emprunt à l'arabe et les processus d'intégration", dans A. Queffélec, F. Benzakour et Y. Cherrad-Benchefra (éds), Le français au Maghreb, Aix-en-Provence: Pub. Université de Provence, 1995, pp. 131-151.

- SMAALI D., Les particularités lexicales du français dans la presse algérienne actuelle, Mémoire de maîtrise, Université de Provence, 1994.

- DEROY L., "Vingt ans après l'Emprunt linguistique: critique et réflexions", Cahiers de l'Institut de Linguistique de Louvain, 6, 1-2, 1980. 
- Malmberg B., Le langage signe de l'humain, Picard, collection Empreinte, Paris, 1979, pp.192-193.

- Morsly, D. Le français dans la réalité algérienne. Thèse de doctorat d'État, Université de Paris V. 1988.

- Fitouri C., Biculturalisme, bilinguisme et éducation, Ed. Delachaux et Niestlé, 1983.

- Mortureux M-F, «La Néologie Lexicale : De L'impasse à L'ouverture », In Langages n ${ }^{\circ}$ 183, 2011.

- Grunig B-N, "Dynamique et lexique », In L'innovation lexicale, Honoré Champion Éditeur, Paris, 2003.

- Nouveau Petit Robert, Dictionnaire alphabétique et analogique de la langue française, version 2.1. Le CD-Rom, 2001.

- Jean Dubois et al, Dictionnaire de linguistique, Larousse, Paris, 2002. 